CARDIOVASCULAR MEDICINE

\title{
Triggering of acute coronary syndromes by physical exertion and anger: clinical and sociodemographic characteristics
}

\author{
P C Strike, L Perkins-Porras, D L Whitehead, J McEwan, A Steptoe
}

See end of article for authors' affiliations

Correspondence to

Correspondence to:
Professor Andrew Steptoe, Department of

Epidemiology and Public Health, University College London, 1-19 Torrington

Place, London WCIE 6BT,

UK; a.steptoe@ucl.ac.uk

Accepted

20 December 2005

Published Online First

6 January 2006

......................

\begin{abstract}
Objective: To investigate the role of vigorous physical exertion and anger as triggers of acute coronary syndromes (ACS) and to identify the clinical and sociodemographic correlates of triggering. Design: Prospective observational clinical cohort study.

Setting: Four coronary care units in the London area.

Patients: 295 men and women with electrocardiographically and biochemically verified ACS.

Main outcome measures: Physical exertion in the $1 \mathrm{~h}$ and anger in the $2 \mathrm{~h}$ before symptom onset were assessed with structured interviews. Control periods were the equivalent hours one day earlier and usual rates over the past six months. Data were analysed by case-crossover methods.

Results: Physical exertion was reported by $10 \%$ and anger by $17.4 \%$ of patients in the hazard period. The risk of ACS onset after physical exertion compared with light or no activity was $3.50195 \%$ confidence interval (CI) 1.37 to 10.6). The risk of onset with anger was $2.06(95 \% \mathrm{Cl} 1.12$ to 3.92$)$. Physical exertion during the hazard period was related to an absence of premonitory symptoms, presentation with an ST elevation myocardial infarction (STEMI), low socioeconomic deprivation and higher future cardiovascular risk. Anger during the hazard period was more common in younger, socioeconomically deprived patients who presented with a STEMI.

Conclusions: Triggers are relevant across the spectrum of ACS. The distinct clinical and sociodemographic factors associated with physical exertion and anger suggest that different pathophysiological processes may be involved.
\end{abstract}

S ome acute coronary syndromes (ACS) appear to be triggered by behavioural or emotional states. ${ }^{12}$ Physical exertion and episodes of anger occurring within $1-2 \mathrm{~h}$ of onset of symptoms have been identified as triggers of acute myocardial infarction (MI). ${ }^{3-6}$ Many studies of triggering were carried out before the development of the modern concept of ACS, encompassing ST segment elevation MI (STEMI), non-ST segment elevation MI (NSTEMI) and unstable angina (UA). ${ }^{7}$ The extent to which physical exertion or anger triggers different types of ACS is not known. Triggering of acute MI by physical exertion has been reported to be more common in men and younger patients in some studies but not others. ${ }^{8-10}$ An association between triggering by anger and low socioeconomic status (SES) was observed in the Onse ${ }^{11}$ but not in the Stockholm Heart Epidemiology Program (SHEEP) study. ${ }^{6}$ The objective of this study was to assess the occurrence of triggering by physical exertion or anger in patients experiencing a broad spectrum of ACS, and to identify the clinical and sociodemographic characteristics of patients who experience triggering.

\section{METHODS}

\section{Participants}

Participants were 295 patients admitted with ACS to four hospitals in the London area. Patients were included in the study on the following criteria: a diagnosis of ACS based on the presence of chest pain plus verification by diagnostic ECG changes (namely, new ST elevation $>0.2 \mathrm{mV}$ in two contiguous leads in leads $\mathrm{V} 1, \mathrm{~V} 2$ or $\mathrm{V} 3$ and $>0.1 \mathrm{mV}$ in two contiguous other leads, ST depression $>0.1 \mathrm{mV}$ in two contiguous leads in the absence of any QRS confounders, and new left branch block or dynamic T wave inversion in more than one lead), or troponin $\mathrm{T}$ measurement $>0.1 \mu \mathrm{g} / \mathrm{l}$ or a creatine kinase measurement more than twice the upper range of normal for the measuring laboratory. ${ }^{7}$ Other inclusion criteria were ability to recall a specific time of onset of symptoms, absence of co-morbid conditions that can influence either symptom presentation or mood (such as severe psychiatric illness, unexplained anaemia, ongoing infection or inflammatory conditions, neoplasia and renal failure), other conditions that can cause troponin positivity ${ }^{12}$ and ability to complete the research interview in English. Patients younger than 18 or older than 90 years were excluded. Data were collected between 2001 and 2004. The study was approved by the Joint University College London/ University College London Hospital Ethics Committee and the ethics committees of participating hospitals.

\section{Clinical measures}

Information was obtained from medical notes about cardiovascular history, clinical factors during admission, and management. Admission ECGs were reviewed by a cardiologist and scrutinised for such presentation as STEMI or NSTEMI. Discharge ECGs were analysed to assess the evolution of $\mathrm{Q}$ or non-Q wave infarction. The highest troponin $\mathrm{T}$ ( $86 \%$ of patients) and creatine kinase $(79 \%$ of patients) concentrations measured during the admission were recorded. We also computed composite risk scores based on the algorithm developed in the Global Registry of Acute

Abbreviations: ACS, acute coronary syndromes; GRACE, Global Registry of Acute Coronary Events; MI, myocardial infarction; NSTEMI, non-ST segment elevation myocardial infarction; PTCA, percutaneous transluminal coronary angioplasty; SES, socioeconomic status; SHEEP, Stockholm Heart Epidemiology Program; STEMI, ST segment elevation myocardial infarction; $U A$, unstable angina 
Coronary Events (GRACE) study. ${ }^{13}$ This uses nine criteria (age, history of congestive heart failure, history of MI, systolic blood pressure and heart rate on admission, ST segment depression, initial serum creatinine, raised cardiac enzymes and no in-hospital percutaneous coronary intervention) to define risk of six-month postdischarge death applicable to all types of ACS.

\section{Procedure and assessment of triggers}

Patients were invited to take part as soon as possible after admission. The study was explained orally and a patient information sheet was provided. Written consent was obtained. A total of 375 patients were potentially eligible for the study; of these, $48(12.8 \%)$ were discharged or transferred to other hospitals before the interview could take place and a further $32(8.5 \%)$ declined participation. The interviews were carried out an average of 2.56 (SD 1.5) days after admission, with $95 \%$ being completed within five days of admission.

The structured triggering interview was based on the procedures used in the Onset and SHEEP studies. ${ }^{34614}$ Patients were questioned in detail about the circumstances surrounding the onset of acute symptoms. Vigorous physical exertion was defined as activity of at least six metabolic equivalents, as used in earlier studies. ${ }^{3514}$ In the light of previous literature, we specifically focused on physical exertion within $1 \mathrm{~h}$ of symptom onset ${ }^{3514}$ and anger within $2 \mathrm{~h}$ of symptom onset. ${ }^{4}{ }^{6}$ For the pair-matched analysis, data were obtained for the corresponding period $24 \mathrm{~h}$ before symptom onset. Additionally, we inquired about usual rates of vigorous physical exertion and anger by assessing the frequency and duration of exposure over the previous six months, by using methods applied in previous studies. ${ }^{36}$

\section{Other measures}

Patients were asked whether they had experienced any relevant symptoms (particularly chest pain) over the four days preceding acute onset and were subsequently categorised according to whether they had premonitory symptoms. Information concerning education, smoking, alcohol consumption and habitual physical activity was collected by questionnaire. SES was assessed with a deprivation index of access to resources on the basis of four criteria: living in a crowded household (defined as one or more people to a room), renting as opposed to owning a home, not having use of a motor vehicle (car or van), and living on state benefits. ${ }^{15}$ Participants were classified as having a low deprivation (negative on all items), medium deprivation (one positive) and high deprivation (2-4 positive).

\section{Statistical analysis}

The study used a case-crossover design to assess the risk of exposure to physical exertion and anger during a "hazard period" before the onset of ACS. ${ }^{16}$ Onset was defined as the time at which the symptoms that led to admission began. Risk of exposure to anger and physical exertion during the hazard period was calculated relative to two control periods: a $\mathrm{l} \mathrm{h}$ (exertion) or $2 \mathrm{~h}$ (anger) period $24 \mathrm{~h}$ before symptom onset (pair-matched analysis) and the previous six months. ${ }^{17}$ The pair-matched case-crossover design is analogous to a matched-pair case-control design, but with participants serving as their own controls. Frequencies of discordant and concordant exposure in the control and hazard periods were therefore used to derive an odds ratio with 95\% confidence intervals (CIs). The usual frequency approach was used to compare exposure during the hazard period with exposure during the previous six months. This is akin to a series of retrospective matched follow-up studies of $\mathrm{n}=1$, where participants act as their own controls. Data for each

\begin{tabular}{|c|c|}
\hline Men/women & $231 / 64$ \\
\hline Age (years) & $60.4(11.6)$ \\
\hline Ethnicity: white & $245(83.1 \%)$ \\
\hline Marital status: married & 191 (64.7\%) \\
\hline \multicolumn{2}{|l|}{ Educational qualifications } \\
\hline None & $139(47.1 \%)$ \\
\hline Primary & $66(22.4 \%)$ \\
\hline Secondary & $90(30.5 \%)$ \\
\hline Paid employment & 158 (53.7\%) \\
\hline \multicolumn{2}{|l|}{ Deprivation index } \\
\hline Low & 132 (44.7\%) \\
\hline Medium & $77(26.1 \%)$ \\
\hline High & $86(29.2 \%)$ \\
\hline Body mass index $\left(\mathrm{kg} / \mathrm{m}^{2}\right)$ & $27.1(4.5)$ \\
\hline \multicolumn{2}{|l|}{ Smóking status } \\
\hline Never & $65(22.0 \%)$ \\
\hline Former smoker & $108(36.6 \%)$ \\
\hline Current smoker & $122(41.4 \%)$ \\
\hline Alcohol drinkers & $188(63.9 \%)$ \\
\hline \multicolumn{2}{|l|}{ Physical activity } \\
\hline None & $182(62.1 \%)$ \\
\hline Low & $60(20.5 \%)$ \\
\hline$>2$ times/week & $51(17.4 \%)$ \\
\hline Diabetes & $39(13.2 \%)$ \\
\hline Hypertension & $131(44.4 \%)$ \\
\hline Previous myocardial infarction & $32(10.8 \%)$ \\
\hline Preadmission $\beta$ blockers & $52(17.7 \%)$ \\
\hline Preadmission statins & $43(14.6 \%)$ \\
\hline Preadmission aspirin & $61(20.7 \%)$ \\
\hline \multicolumn{2}{|l|}{ Time of onset } \\
\hline $0000-0600$ & $62(21.0 \%)$ \\
\hline $0600-1200$ & $96(32.5 \%)$ \\
\hline $1200-1800$ & $84(28.5 \%)$ \\
\hline $1800-2400$ & $53(18.0 \%)$ \\
\hline Woke up with symptoms & $29(9.8 \%)$ \\
\hline \multicolumn{2}{|l|}{ Day of week } \\
\hline Weekday & 197 (66.8\%) \\
\hline Weekend & $98(33.2 \%)$ \\
\hline Premonitory symptoms ( 4 days) & $134(45.4 \%)$ \\
\hline \multicolumn{2}{|l|}{ Acute coronary syndrome type } \\
\hline STEMI & 200 (67.8\%) \\
\hline NSTEMI/UA & $95(32.3 \%)$ \\
\hline ST segment depression & $36(12.2 \%)$ \\
\hline$Q$ wave & $131(50.2 \%)$ \\
\hline Non- $Q$ wave & $130(49.8 \%)$ \\
\hline No of vessels diseased & $1.78(0.8)$ \\
\hline Heart failure & $23(7.8 \%)$ \\
\hline Arrhythmia & $25(8.5 \%)$ \\
\hline Troponin T ( $\mu \mathrm{g} / \mathrm{l})(\mathrm{n}=253)$ & $4.03(5.7)$ \\
\hline Creatine kinase (U/I) (n=233) & $1286.3(1395)$ \\
\hline \multicolumn{2}{|l|}{ Treatment } \\
\hline Medical & $80(28.7 \%)$ \\
\hline PTCA & $159(57.0 \%)$ \\
\hline CABG & $40(14.3 \%)$ \\
\hline GRACE risk score & $93.0(27.1)$ \\
\hline \multicolumn{2}{|c|}{$\begin{array}{l}\text { Data are mean (SD) or number (\%). } \\
\text { CABG, coronary artery bypass grafting; GRACE, Global } \\
\text { Registry of Acute Coronary Events; NSTEMI, non-ST } \\
\text { elevation myocardial infarction; PTCA, percutaneous } \\
\text { transluminal coronary angioplasty; STEMI, ST elevation } \\
\text { myocardial infarction; UA, unstable angina. }\end{array}$} \\
\hline
\end{tabular}

Table 2 Physical exertion and anger during the hazard and control periods

\begin{tabular}{lll}
\hline & $\begin{array}{l}\text { Physical } \\
\text { exertion }\end{array}$ & Anger \\
\hline Exposed during hazard period only & $21(8.1 \%)$ & $36(14.2 \%)$ \\
Exposed during control period only & $6(2.3 \%)$ & $17(6.7 \%)$ \\
Exposed during hazard and control & $5(1.9 \%)$ & $8(3.2 \%)$ \\
periods & $226(87.6 \%)$ & $192(75.9 \%)$ \\
Not exposed & & \\
\hline
\end{tabular}




\begin{tabular}{|c|c|c|c|c|}
\hline & $\begin{array}{l}\text { Exertion trigger } \\
\text { group }\end{array}$ & $\begin{array}{l}\text { Comparison } \\
\text { group }\end{array}$ & $\begin{array}{l}\text { Univariate } \\
\text { p value }\end{array}$ & Odds ratio* $(95 \% \mathrm{Cl})$ \\
\hline \multicolumn{5}{|l|}{ Time of onset: } \\
\hline $0000-0600$ & 0 & $44(18.4 \%)$ & \multirow[t]{4}{*}{0.007} & \\
\hline $0600-1200$ & $8(30.8 \%)$ & $79(33.1 \%)$ & & \\
\hline $1200-1800$ & $15(57.7 \%)$ & $68(28.5 \%)$ & & \\
\hline $1800-2400$ & $3(11.5 \%)$ & $48(20.1 \%)$ & & \\
\hline \multicolumn{5}{|l|}{ Deprivation } \\
\hline Low & $17(65.4 \%)$ & $98(41.0 \%)$ & \multirow[t]{3}{*}{0.022} & 1 \\
\hline Medium & $5(19.2 \%)$ & $67(28.0 \%)$ & & $0.44(0.15$ to 1.25$)$ \\
\hline High & $4(15.4 \%)$ & $74(31.0 \%)$ & & $0.31(0.10$ to 0.97$)$ \\
\hline \multicolumn{5}{|c|}{ Acute coronary syndrome type } \\
\hline NSTEMI/UA & $5(19.2 \%)$ & $83(37.2 \%)$ & \multirow[t]{3}{*}{0.019} & 1 \\
\hline Non-Q STEMI & $5(19.2 \%)$ & $57(25.6 \%)$ & & $1.44(0.40$ to 5.27$)$ \\
\hline$Q$ wave STEMI & $16(61.5 \%)$ & $83(37.2 \%)$ & & $3.06(1.07$ to 8.78$)$ \\
\hline \multicolumn{5}{|c|}{ Premonitory symptoms } \\
\hline Yes & $3(11.5 \%)$ & $116(48.5 \%)$ & \multirow[t]{2}{*}{0.001} & 1 \\
\hline No & $23(88.5 \%)$ & $123(51.5 \%)$ & & $7.41(2.16$ to 25.4$)$ \\
\hline \multicolumn{5}{|c|}{ Troponin T quartile ( $\mu \mathrm{g} / \mathrm{l})$} \\
\hline $1(<0.56)$ & $4(18.2 \%)$ & $56(27.9 \%)$ & \multirow[t]{4}{*}{0.014} & \\
\hline$(0.56-1.85)$ & $4(18.2 \%)$ & $54(26.9 \%)$ & & $(0.24$ to 4.25$)$ \\
\hline $3(1.86-5.50)$ & $2(9.1 \%)$ & $50(24.9 \%)$ & & $0.57(0.10$ to 3.29$)$ \\
\hline $4(>5.50)$ & $12(54.5 \%)$ & $41(20.4 \%)$ & & $3.60(1.18$ to 13.2$)$ \\
\hline GRACE risk score & $102.0(27.9)$ & $94.2(27.1)$ & 0.005 & \\
\hline
\end{tabular}

person were pooled, with the person as the stratifying variable, and analysed by using methods for sparse data in each stratum on the basis of a Mantel-Haenszel calculation of relative risk, estimated as the ratio between observed and expected odds. ${ }^{18}$ Relative risks were calculated by conditional logistic regression adjusted for age and sex.

\section{RESULTS}

Participants were predominantly men, white and with limited education (table 1). Men were significantly younger than women on average and were more likely to be in paid employment $(p<0.001)$. Men and women did not differ significantly in medication, clinical presentation or other cardiovascular risk factors.

Symptoms were more likely to have started between 0600 and 1800 than in the evening or early morning $(p<0.001)$, with no difference between sexes. Twenty nine patients were woken with acute symptoms after sleeping for more than $2 \mathrm{~h}$, so they could not provide trigger information. Data for the pair-matched control periods were not available for a further seven (physical exertion) and 13 (anger) participants. Admissions were equally distributed across the year, and the distribution of events occurring on week days and weekends did not differ from chance. Premonitory symptoms over the previous four days were reported by 134 (45.4\%) patients. The average number of diseased vessels was slightly greater in men $(p<0.05)$. Men were more likely than women to receive percutaneous transluminal coronary angioplasty (PTCA) or coronary artery bypass graft $(\mathrm{p}=0.035)$.

\section{Triggering by physical exertion}

Twenty six $(10 \%)$ of the patients who provided information about the hazard period reported vigorous physical exertion in the $1 \mathrm{~h}$ before symptom onset. Activities included heavy gardening, lifting during house removal and gym workouts. Table 2 details the numbers of patients who reported vigorous exertion during the hazard and control periods. By the pairmatched case-crossover approach, the odds of ACS starting after physical exertion compared with light or no activity were 3.50 (95\% CI 1.37 to 10.6). When compared with the previous six months, the relative risk was 8.62 (95\% CI 5.81 to 12.78 ).

Table 3 summarises the clinical and sociodemographic characteristics associated with physical exertion in the hazard period. There was no association with age, sex, ethnicity, preadmission cardiovascular risk factors, number of diseased arteries or cardiac history. Time of day had a pronounced effect; no patients whose symptoms started between midnight and 0600 reported exertion in the hazard period, and the rate was highest $(18.1 \%)$ among patients with symptom onset in the afternoon. Adjusted odds ratios have not been computed, as the reference group (midnight to 0600 ) had no cases. An inverse relationship with social deprivation was also observed. More socially deprived participants were less likely than affluent patients to be physically active in the hazard period.

Patients whose ACS was apparently triggered by physical exertion were more likely to develop a Q wave STEMI $(p=0.019)$. A strong association with lack of premonitory symptoms was evident, with $15.8 \%$ of patients with no prior symptoms being physically active during the hazard period compared with $2.5 \%$ of those who had premonitory symptoms $(\mathrm{p}<0.001)$. Physical exertion during the hazard period was associated with greater troponin release $(p=0.014)$ and with a raised GRACE risk score $(p=0.024)$ in comparison with patients who were sedentary before symptom onset.

\section{Triggering by anger}

Forty four patients (17.4\%) reported anger in the $2 \mathrm{~h}$ before symptom onset. Episodes included arguments with neighbours, anger during commuting and family conflict. Triggering by physical exertion and triggering by anger did not overlap significantly, as only four participants reported both types of trigger. The odds ratio for onset of ACS after anger compared with no anger in the pair-matched analysis was 2.06 (95\% CI 1.12 to 3.92). The relative risk of anger occurring in the hazard period compared with the previous six months was 7.30 (95\% CI 5.22 to 10.19).

Anger in the $2 \mathrm{~h}$ before symptom onset was not associated with sex, ethnicity, preadmission cardiovascular risk factors 


\begin{tabular}{|c|c|c|c|c|}
\hline & $\begin{array}{l}\text { Anger trigger } \\
\text { group }\end{array}$ & $\begin{array}{l}\text { Comparison } \\
\text { group }\end{array}$ & $\begin{array}{l}\text { Univariate } \\
\text { p value }\end{array}$ & Odds ratio* $(95 \% \mathrm{Cl})$ \\
\hline \multicolumn{5}{|l|}{ Age } \\
\hline$\leqslant 50$ & $15(34.1 \%)$ & $42(19.5 \%)$ & 0.024 & 1 \\
\hline $51-60$ & $14(31.8 \%)$ & $67(31.2 \%)$ & & 0.58 (0.25 to 1.32 ) \\
\hline $61-70$ & $8(18.2 \%)$ & $48(22.3 \%)$ & & $0.45(0.17$ to 1.18$)$ \\
\hline$\geqslant 71$ & $7(15.9 \%)$ & $58(27.0 \%)$ & & $0.32(0.11$ to 0.88$)$ \\
\hline \multicolumn{5}{|l|}{ Deprivation } \\
\hline Low & $14(31.8 \%)$ & $99(46.0 \%)$ & 0.040 & 1 \\
\hline Medium & $12(27.3 \%)$ & $59(27.4 \%)$ & & $1.41(0.60$ to 3.27$)$ \\
\hline High & $18(40.9 \%)$ & $57(26.5 \%)$ & & $2.14(1.00$ to 4.66$)$ \\
\hline \multicolumn{5}{|c|}{ Acute coronary syndrome type } \\
\hline NSTEMI/UÁ & $8(19.0 \%)$ & $80(39.6 \%)$ & 0.025 & 1 \\
\hline Non-Q STEMI & $15(35.7 \%)$ & $45(22.8 \%)$ & & 3.23 (1.26 to 8.28$)$ \\
\hline$Q$ wave STEMI & $19(45.2 \%)$ & $76(37.6 \%)$ & & $2.60(1.06$ to 6.39$)$ \\
\hline
\end{tabular}

or cardiac history. But anger preceding ACS onset was more common in younger patients $(p=0.024)$ and in those of lower SES as defined by deprivation $(\mathrm{p}=0.04)$ : $24 \%$ of the most deprived group reported anger during the hazard period compared with $12.4 \%$ of the least deprived (table 4 ). Anger during the hazard period was also more common in patients presenting with STEMI than with NSTEMI/UA ( $p=0.028)$. Triggering by anger was not related to the presence of premonitory symptoms, time of onset, troponin release or GRACE risk score.

\section{Comparison of patients with exertion and anger triggers}

Patients reporting physical exertion and patients reporting anger in the hazard period were directly compared, omitting four participants who experienced both triggers. There were no differences in sex, age or number of diseased vessels

Table 5 Comparison of patients with physical exertion and anger triggers

\begin{tabular}{|c|c|c|c|}
\hline & $\begin{array}{l}\text { Exertion } \\
\text { trigger } \\
\text { ( } n=22 \text { ) }\end{array}$ & $\begin{array}{l}\text { Anger } \\
\text { trigger } \\
(n=40)\end{array}$ & $\begin{array}{l}\mathrm{p} \\
\text { Value }\end{array}$ \\
\hline Proportion of men & $20(90.9 \%)$ & $31(77.5 \%)$ & 0.30 \\
\hline Age (years) & $59.5(11.2)$ & $56.9(11.6)$ & 0.40 \\
\hline \multicolumn{4}{|l|}{ Deprivation index } \\
\hline Low & $15(68.2 \%)$ & $12(30.0 \%)$ & \multirow[t]{3}{*}{0.002} \\
\hline Medium & $5(22.7 \%)$ & $12(30.0 \%)$ & \\
\hline High & $2(9.1 \%)$ & $16(40.0 \%)$ & \\
\hline \multicolumn{4}{|l|}{ Timing of onset } \\
\hline $0000-0600$ & 0 & $6(15.0 \%)$ & \multirow[t]{4}{*}{0.006} \\
\hline $0600-1200$ & $6(27.3 \%)$ & $12(30.0 \%)$ & \\
\hline $1200-1800$ & $14(63.6 \%)$ & $11(27.5 \%)$ & \\
\hline $1800-2400$ & $2(9.1 \%)$ & $11(27.5 \%)$ & \\
\hline Premonitory symptoms present & $2(9.1 \%)$ & $18(45.0 \%)$ & 0.004 \\
\hline Current smoker & $11(50.0 \%)$ & 19. $(47.5 \%)$ & 0.85 \\
\hline Diabetes & $5(22.7 \%)$ & $7(17.5 \%)$ & 0.74 \\
\hline Hypertension & $6(27.3 \%)$ & $22(55.0 \%)$ & 0.061 \\
\hline Previous myocardial infarction & $3(1.6 \%)$ & $4(10.0 \%)$ & 0.69 \\
\hline \multicolumn{4}{|c|}{ Troponin T quartile $(n=52)(\mu \mathrm{g} / \mathrm{l})$} \\
\hline $1(<0.56)$ & $2(10.5 \%)$ & $8(24.2 \%)$ & \multirow[t]{4}{*}{0.034} \\
\hline $2(0.56-1.85)$ & $4(21.1 \%)$ & $7(21.2 \%)$ & \\
\hline $3(1.86-5.50)$ & $1(5.3 \%)$ & $9(27.3 \%)$ & \\
\hline $4(>5.50)$ & $12(63.2 \%)$ & $9(27.3 \%)$ & \\
\hline \multicolumn{4}{|l|}{ Acute coronary syndrome type } \\
\hline NSTEMI/UA & $5(22.7 \%)$ & $8(21.1 \%)$ & \multirow[t]{3}{*}{0.28} \\
\hline Non-Q STEMI & $4(18.2 \%)$ & $14(36.8 \%)$ & \\
\hline Q wave STEMI & $13(59.1 \%)$ & $16(42.1 \%)$ & \\
\hline No of vessels diseased & $1.56(0.9)$ & $1.80(0.9)$ & 0.33 \\
\hline GRACE risk score & $102.1(28.8)$ & $85.1(27.4)$ & 0.034 \\
\hline
\end{tabular}

GRACE, Global Registry of Acute Coronary Events; NSTEMI, non-ST elevation myocardial infarction; STEMI, ST elevation myocardial infarction; UA, unstable angina. (table 5). Patients who reported anger during the hazard period were more likely to be socioeconomically deprived $(p=0.006)$ and to have experienced premonitory symptoms over the previous four days $(p=0.004)$. Physical exertion during the hazard period was associated with onset in the afternoon $(\mathrm{p}=0.002)$, higher troponin concentrations $(p=0.034)$ and raised GRACE risk scores $(p=0.034)$. No other differences in lifestyle factors, cardiovascular history or presentation were observed, although more of those in the anger than in the exertion trigger groups tended to have hypertension (non-significant).

\section{DISCUSSION}

The purposes of this investigation were to discover whether the behavioural and emotional stimuli previously identified as triggers of acute MI are relevant to the broader spectrum of ACS, and to assess the clinical and sociodemographic characteristics of patients who report possible triggers during the hours preceding symptom onset. We used case-crossover methods to analyse these transient acute effects. The advantages of the case-crossover method are that patients serve as their own referents, leading to efficient control of fixed potential confounders and unmeasured personal attributes, and an ability to take habitual rates of exposure into account. ${ }^{16}$

The proportion of patients who reported vigorous physical exertion in the $\mathrm{l} \mathrm{h}$ preceding symptom onset was $10 \%$. Previous studies of acute MI have reported heavy exertion in $4.4 \%, 6.4 \%$ and $7.1 \%,{ }^{35}{ }^{14}$ with moderate or vigorous physical activity in $18.7 \%$ and $27.1 \% .^{14}{ }^{19}$ The odds ratio of 3.50 from the pair-matched analysis compares with $4.2^{14}$ and $5.6^{3}$ in previous studies. The relative risk versus habitual exercise levels of 8.62 compares with risks ranging from 3.3-10.1. ${ }^{1}$ The prevalence of anger over the $2 \mathrm{~h}$ before symptom onset $(17.4 \%)$ was greater than that reported in the Onset and SHEEP studies, both of which had a prevalence of less than $2.5 \% .{ }^{4}$ This is because of our use of broader inclusion criteria for anger episodes. Previous studies have analysed only high levels of anger; when the corresponding criterion was applied to the present data set, prevalence of anger fell to $3.5 \%$, but the number of cases was too small for further analysis. The relative risk for anger triggering over the previous six months of 7.30 compares with 5.7 in the SHEEP study. ${ }^{6}$ The higher relative risks when the hazard periods were compared with usual rates of exposure rather than the pair-matched periods is probably due to less accurate recall of habitual rates of exercise and mood, in comparison with recall of specific time periods.

The patients with ACS in this study sample were relatively young, with only moderate risk profiles as measured with the 
GRACE index..$^{13}$ The low levels of education are comparable with attainment in a national sample of men and women of this age in England. ${ }^{20}$ There was also a higher proportion of STEMI than of NSTEMI/UA than that reported in recent studies, ${ }^{21}$ probably a result of the requirement that patients could recall a specific time of onset. We did not observe any sex difference in presentation with STEMI and NSTEMI/UA, but as in previous studies women had fewer diseased coronary arteries. ${ }^{22}$ Most patients underwent PTCA, but a higher proportion of women than men were managed medically, as has previously been described in the literature. ${ }^{23}$

We identified distinctive profiles of patients who reported triggering by physical exertion and anger. Physical exertion in the $\mathrm{l}$ h before symptom onset was associated with absence of premonitory symptoms, higher SES as measured by the deprivation index, development of a Q wave STEMI and a higher clinical risk score. In contrast, anger during the hazard period was associated with lower SES, younger age and presentation with STEMI, and had no relationship with premonitory symptoms. Direct comparison of the patients who reported either exertion or anger during the hazard period confirmed that exertion was associated with less socioeconomic deprivation, fewer premonitory symptoms, greater troponin release and higher clinical risk scores than was anger. The association between lack of premonitory symptoms and triggering by physical exertion has been described before ${ }^{14}$ and is perhaps not surprising, as people who experience symptoms over several days are unlikely to voluntarily exert themselves physically. We did not observe a protective effect of regular physical activity in this study, ${ }^{35}$ and this may have been due to the small sample size or to the sedentary nature of the population.

Both types of triggering were more commonly associated with STEMI than with other forms of ACS. Nevertheless, $5.7 \%$ of patients admitted with NSTEMI/UA reported physical exertion and $9.1 \%$ experienced anger during the hazard period, so triggering may occur across the spectrum of ACS. An interesting difference emerged with respect to the nature of the STEMI, with associations between the development of a Q wave infarction and physical exertion but not anger. This pattern, coupled with the relationship between physical exertion, troponin release and GRACE risk scores, suggests that this group of patients has greater myocyte damage and a raised risk of future morbidity. Silent ischaemia during ambulatory ECG monitoring has also been associated with heavy physical exertion. ${ }^{24}$

The mechanisms underlying these patterns of triggering are not fully understood. Physical exertion elicits an acute increase in sympathetic activity, release of catecholamines and haemodynamic responses that may stimulate plaque rupture. Catecholamines stimulate surface expression of adhesion molecules, and the concentration of interleukin 6 in the circulation rises notably. ${ }^{25}$ Acute mental stress also stimulates inflammatory cytokines, lymphocyte adhesion molecule expression and platelet activation. ${ }^{26} 27$ Platelet reactivity to mental stress has been positively correlated with hostile personality traits, ${ }^{28}$ and this may contribute to the association with anger. Although anger triggering was related to presentation with STEMI, however, it was not associated with the development of Q wave infarction, heart failure or the magnitude of troponin T release. This suggests that anger may have transiently influenced physiology and implicates a possible role of vascular spasm. Boltwood et al ${ }^{29}$ reported that anger recall during catheterisation induced coronary vasoconstriction in stenosed vessels, and this phenomenon may translate into clinical risk of ACS. Emotional stress has been shown in ambulatory monitoring studies to induce transient myocardial ischaemia. ${ }^{24}$ Another relevant mechanism is disturbance of cardiac autonomic function leading to arrhythmia. ${ }^{30}$
Anger in the hours immediately preceding symptom onset was associated with younger age and greater socioeconomic deprivation. The age effect suggests that people who are prone to anger are less likely to survive into old age. A relationship between anger triggering and lower SES defined by education was described in the Onset study. ${ }^{11}$ This result is consistent with the notion that emotional factors are responsible in part for the socioeconomic gradient in risk of coronary heart disease.

This study has some limitations. The sample size was relatively small and prevented us from dividing out NSTEMI from UA. Exclusion of patients with co-morbidities that can affect symptom presentation, mood or cardiac enzymes led to restriction of the sample to people with relatively low risk. Patients' retrospective reports of their experiences before symptom onset may be biased or influenced by the salience of the situation, leading to overestimation during the hazard period. ${ }^{1}$ The strengths of the investigation include assessment of different types of ACS, characterisation of patients in terms of multivariate clinical risk, and measurement of troponin in the majority of patients. The results indicate that triggering by exertion and anger is relevant not only to acute MI but also to other presentations across the range of ACS. The distinctive clinical and sociodemographic profiles of triggering by exertion and anger suggest that different patients are at risk from these factors. It remains to be seen whether the pathophysiological processes underlying triggering by physical exertion and anger also vary.

\section{ACKNOWLEDGEMENTS}

We are grateful to Dr Sue Edwards for participation in data collection, Dr Johan Hallqvist and Dr Jette Möller for assistance with the triggering interview and statistical analysis, and the staff and patients of University College Hospital, St George's Hospital, Southend Hospital and Kingston Hospital.

\section{Authors' affiliations}

P C Strike, L Perkins-Porras, D L Whitehead, A Steptoe, Department of Epidemiology and Public Health, University College London, London, UK J McEwan, Department of Medicine, University College London, London, UK

This research was supported by the British Heart Foundation. The funding source has not been involved in the submission of the manuscript or in the decision to publish the data.

Competing interests: None declared.

\section{REFERENCES}

1 Strike PC, Steptoe A. Behavioral and emotional triggers of acute coronary syndromes: a systematic review and critique. Psychosom Med 2005;67:179-86.

2 Servoss SJ, Januzzi JL, Muller JE. Triggers of acute coronary syndromes. Prog Cardiovasc Dis 2002:44:369-80.

3 Mittleman MA, Maclure M, Tofler $\mathrm{GH}$, et al. Triggering of acute myocardial infarction by heavy physical exertion: protection against triggering by regular exertion. Determinants of Myocardial Infarction Onset Study Investigators. N Engl J Med 1993;329:1677-83.

4 Mittleman MA, Maclure M, Sherwood JB, et al. Triggering of acute myocardial infarction onset by episodes of anger. Circulation 1995;92:1720-5

5 Willich SN, Lewis $M$, Lowel $\mathrm{H}$, et al. Physical exertion as a trigger of acute myocardial infarction. Triggers and Mechanisms of Myocardial Infarction Study Group. N Engl J Med 1993;329:1684-90.

6 Möller J, Hallqvist J, Diderichsen F, et al. Do episodes of anger trigger myocardial infarction? A case-crossover analysis in the Stockholm Heart Epidemiology Program (SHEEP). Psychosom Med 1999;61:842-9

7 Anon. Myocardial infarction redefined: a consensus document of The Joint European Society of Cardiology/American College of Cardiology Committee for the redefinition of myocardial infarction. Eur Heart J 2000;21:1502-13.

8 Tofler GH, Stone PH, Maclure M, et al. Analysis of possible triggers of acute myocardial infarction (the MILIS study). Am J Cardiol 1990;66:22-7.

9 Miric D, Eterovic D, Giunio L, et al. Triggers of acute myocardial infarction regarding its site. Int J Cardiol 1997;60:67-71.

10 Stewart RA, Robertson MC, Wilkins GT, et al. Association between activity at onset of symptoms and outcome of acute myocardial infarction. J Am Coll Cardiol 1997;29:250-3. 
11 Mittleman MA, Maclure M, Nachnani M, et al. Educational attainment, anger, and the risk of triggering myocardial infarction onset. Arch Intern Med 1997; 157:769-75.

12 Ammann $\mathbf{P}$, Pfisterer $M$, Fehr $T$, et al. Raised cardiac troponins. BMJ 2004;328:1028-9.

13 Eagle KA, Lim MJ, Dabbous OH, et al. A validated prediction model for all forms of acute coronary syndrome: estimating the risk of 6-month postdischarge death in an international registry. JAMA 2004;291:2727-33.

14 Hallqvist J, Moller J, Ahlbom A, et al. Does heavy physical exertion trigger myocardial infarction? A case-crossover analysis nested in a populationbased case-referent study. Am J Epidemiol 2000;151:459-67

15 Krieger N, Williams DR, Moss NE. Measuring social class in US public health research: concepts, methodologies, and guidelines. Annu Rev Public Health 1997; 18:341-78.

16 Maclure M, Mittleman MA. Should we use a case-crossover design? Annu Rev Public Health 2000;21:193-221.

17 Mittleman MA, Maclure M, Robins JM. Control sampling strategies for casecrossover studies: an assessment of relative efficiency. Am J Epidemiol 1995; 142:91-8.

18 Marshall RJ, Jackson RT. Analysis of case-crossover designs. Stat Med 1993; 12:2333-41

19 Tofler GH, Muller JE, Stone PH, et al. Modifiers of timing and possible triggers of acute myocardial infarction in the Thrombolysis in Myocardial Infarction Phase II (TIMI II) Study Group. J Am Coll Cardiol 1992;20:1049-55.

20 Marmot M, Banks J, Blundell R, et al. Health, wealth and lifestyles of the older population in England. London: Institute of Fiscal Studies, 2003.
21 Fox KA, Goodman SG, Klein W, et al. Management of acute coronary syndromes: variations in practice and outcome: findings from the Global Registry of Acute Coronary Events (GRACE). Eur Heart J 2002;23:1 177-89.

22 Rosengren A, Wallentin L, Gitt AK, et al. Sex, age, and clinical presentation of acute coronary syndromes. Eur Heart J 2004; 25:663-70.

23 Glaser R, Herrmann HC, Murphy SA, et al. Benefit of an early invasive management strategy in women with acute coronary syndromes. JAMA 2002;288:3124-9.

24 Gullette EC, Blumenthal JA, Babyak $M$, et al. Effects of mental stress on myocardial ischemia during daily life. JAMA 1997;277:1521-6.

25 Shephard RJ. Adhesion molecules, catecholamines and leucocyte redistribution during and following exercise. Sports Med 2003;33:261-84

26 Steptoe A, Willemsen G, Owen N, et al. Acute mental stress elicits delayed increases in circulating inflammatory cytokine levels. Clin Sci (Colch) $2001 ; 101: 185-92$

27 Von Kanel R, Mills PJ, Fainman C, et al. Effects of psychological stress and psychiatric disorders on blood coagulation and fibrinolysis: a biobehavioral pathway to coronary artery disease? Psychosom Med 2001;63:531-44.

28 Markovitz JH, Matthews KA, Kriss J, et al. Effects of hostility on platelet reactivity to psychological stress in coronary heart disease patients and in healthy controls. Psychosom Med 1996;58:143-9.

29 Boltwood MD, Taylor CB, Burke MB, et al. Anger report predicts coronary artery vasomotor response to mental stress in atherosclerotic segments. Am J Cardiol 1993:72:1361-5.

30 Hemingway $\mathbf{H}$, Malik M, Marmot $M$. Social and psychosocial influences on sudden cardiac death, ventricular arrhythmia and cardiac autonomic function. Eur Heart J 2001;22:1082-101.

\section{IMAGES IN CARDIOLOGY}

Severe aeroportia in a patient with acute myocardial infarction, complicated by acute ischaemic bowel syndrome

A n 83 year old woman presented with acute anterior myocardial infarction, Killip class I. She received percutaneous coronary intervention for ensuing cardiogenic shock. An intra-aortic balloon pump (IABP) was applied via the right femoral route. The patient's haemodynamics became stable within two hours and all inotropes were discontinued. Further episodes of hypotension, sinus tachycardia, metabolic acidosis and acute abdominal distension developed 12 hours after the intervention. The echocardiogram showed good left ventricular contractility, without cardiac tamponade. IABPrelated aortic haemorrhage was suspected initially. Aggressive combination treatment was implemented with high dose dopamine, norepinephrine, epinephrine and vasopressin. However, the shock persisted. Abdominal computed tomography (CT) did not show a retroperitoneal haematoma but did reveal extensive bowel dilatation and massive air in the intrahepatic (panel A) and spleno-portal vein (aeroportia) (panel B). Air within the intestinal submucosa was also noted (pneumatosis intestinalis) (panel C). The diagnosis of acute ischaemic bowel was confirmed. The patient died 15 hours after the initiation of shock, despite aggressive medical treatment.

Pneumatosis intestinalis is an uncommon but important condition in which gas is found in a linear or cystic form in
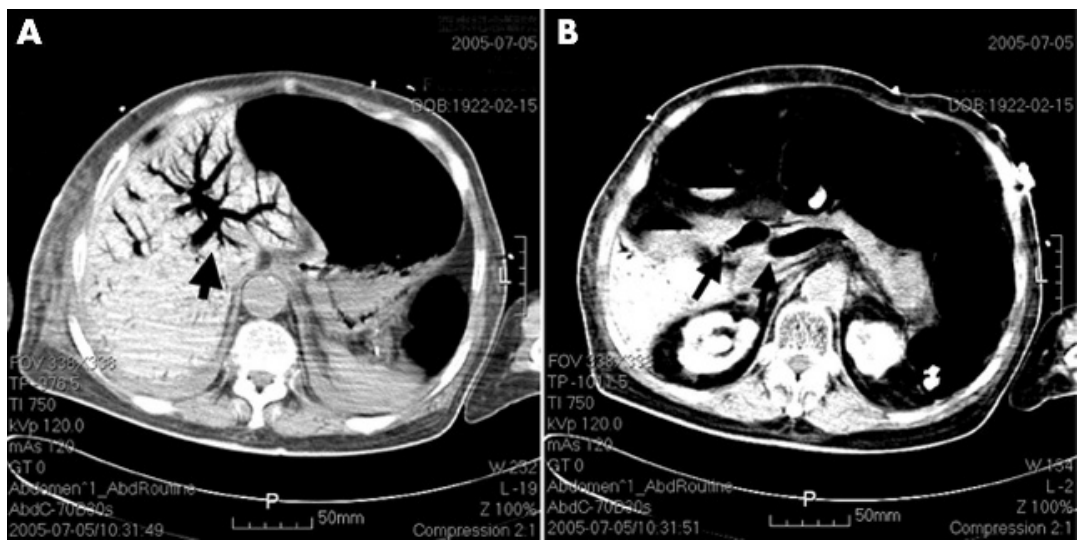

the submucosa or subserosa of the bowel wall. Gas accumulation in the portomesenteric veins (aeroportia) is even more rare. The presence of pneumatosis intestinalis and aeroportia caused by bowel ischaemia does not generally predict transmural bowel infarction, because they may be observed in patients with only partial ischaemic bowel wall damage. The clinical outcome of patients with bowel ischaemia with these CT findings seems to depend mainly on the severity and extent of their underlying disease.

H-T Hsin, J-J Hwang, H-J Lo cv@mail.femh.org.tw

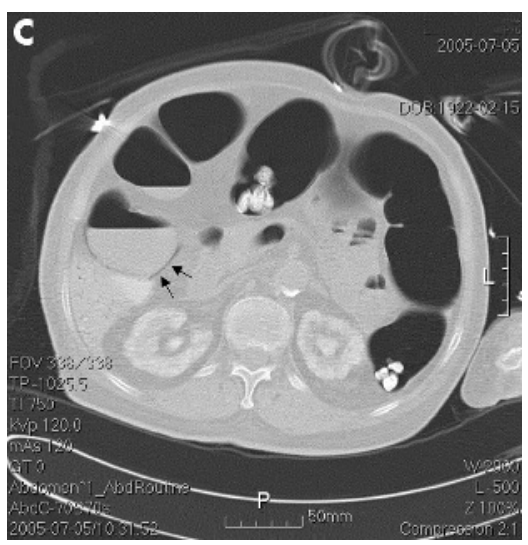

Competing interest statement: There was no competing interest to declare. 